\title{
Interventions for the management of substance use disorders: an overview
}

Richard A. Rawson ${ }^{7}$ and Nicolas Clark ${ }^{2}$

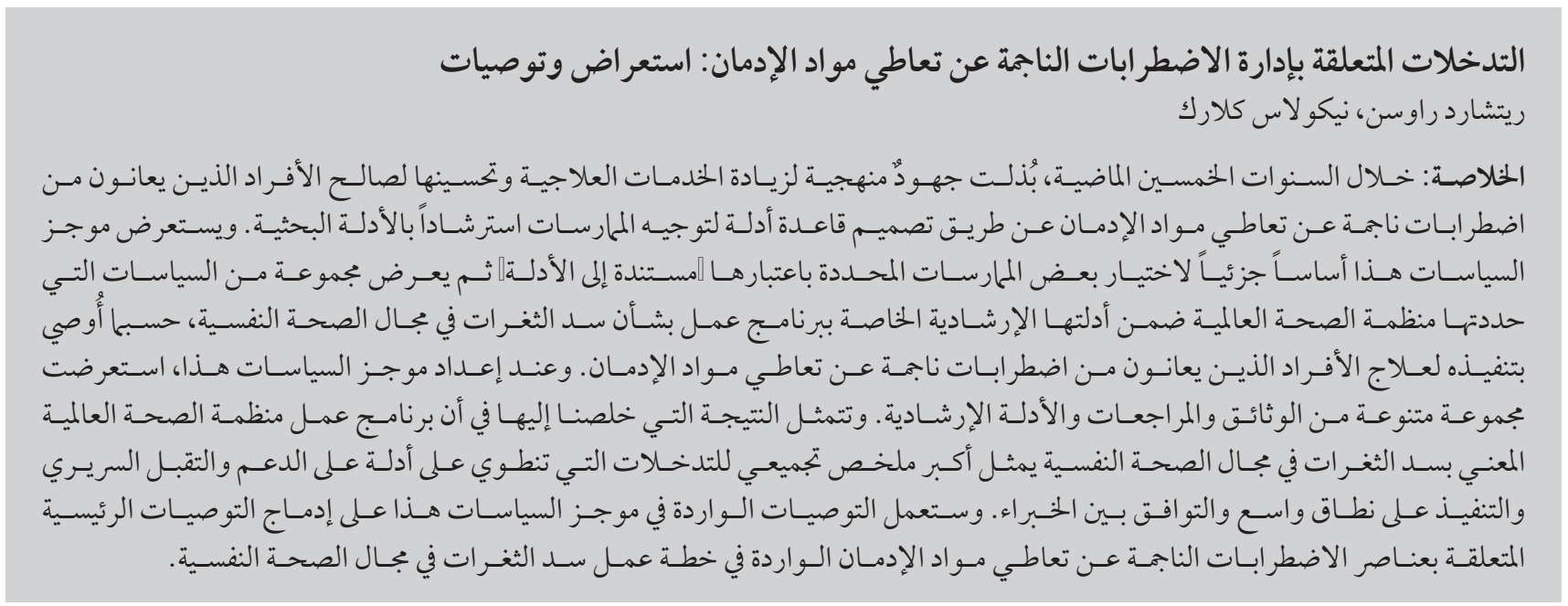

ABSTRACT For the past 50 years, there has been a systematic effort to expand and improve treatment services for individuals with substance use disorders by developing an evidence base to guide practice. This policy brief aims to review the available interventions for the management of substance use disorders, examine the evidence base for these interventions, including the WHO Mental Health Gap Action Programme intervention guide, and make recommendations related to the treatment of substance use disorders. In the development of this policy brief, numerous documents were reviewed. The WHO Mental Health Gap Action Programme intervention guide offers the most well synthesized summary of interventions that have evidence of support, clinical acceptance, extensive implementation and expert consensus. The recommendations within this policy brief incorporate the core recommendations on substance use disorder elements included in the mhGAP intervention guide.

\section{Interventions pour la prise en charge des troubles liés à la consommation de substances psychoactives : examen et recommandations}

RÉSUMÉ Au cours des 50 dernières années, des efforts systématiques ont été déployés pour étendre et améliorer les services de traitement des individus souffrant de troubles liés à la consommation de substances psychoactives, par le biais de l'élaboration d'une base de données factuelles visant à orienter les pratiques. La présente note d'orientation a pour objectif de passer en revue les interventions disponibles pour la prise en charge des troubles liés à la consommation de substances psychoactives, d'examiner les bases factuelles pour ces interventions dont le guide d'intervention du Programme d'action de l'OMS : Combler les lacunes en santé mentale (mhGAP), et d'effectuer des recommandations concernant le traitement de ces troubles. Au cours de l'élaboration de cette note d'orientation, divers documents ont été examinés. Le guide d'intervention du Programme mhGAP de l'OMS offre la meilleure synthèse d'interventions fournissant des informations sur le soutien dont elles bénéficient, l'acceptation clinique, une mise en œuvre étendue, et les consensus d'experts. Les recommandations de la note d'orientation intègrent les recommandations principales portant sur les éléments relatifs aux troubles liés à la consommation de substances psychoactives compris dans le guide d'intervention du Programme mhGAP.

${ }^{\prime}$ Center for Behavior and Health, Department of Psychiatry, University of Vermont, Burlington, Vermont, United States of America (Correspondence to: Richard A. Rawson: rrawson@mednet.ucla.edu). ${ }^{2}$ Department of Mental Health and Substance Abuse, World Health Organization, Geneva, Switzerland.

Received: 19/05/16; accepted: 09/04/17 


\section{Introduction}

This policy brief aims to review the available interventions for the management of substance use disorders, examine the evidence for these interventions including the WHO Mental Health Gap Action Programme (mhGAP) intervention guide, and make recommendations related to the treatment of substance use disorders.

\section{Evidence-based practices}

While there has been a large amount of research on the treatment of substance use disorders, there has been considerable controversy about what constitutes an "evidence-based practice" and which of these practices should be applied in "real world" treatment settings. The importance of translating scientific advances in disease-specific interventions into clinical practice has been emphasized throughout the health care system, largely stemming from the consistent observation of a wide gap between research and practice (1). As a move toward "evidence-based practice" has permeated health care systems and policy, several working groups in the field of addiction treatment have considered ways to align with this initiative. In the field of addiction, however, consensus regarding the optimal procedures for identifying practices with sufficient empirical foundation to be considered "evidence-based" has not yet been reached.

\section{What is evidence-based practice?}

Adapting Sackett et al.'s definition (2), evidence-based practice is characterized as "the integration of best research evidence with clinical expertise and patient values". The most debated components of this definition are the concepts of i) best research evidence and ii) clinical expertise.

\section{Best research evidence}

Although it has been appropriately noted that the definition of "best research evidence" depends upon the nature of the clinical question (e.g. etiological questions versus identification of the most efficacious treatment for a particular disease) (3), descriptions of this concept, to date, uniformly acknowledge a variety of sources from which data can be brought to bear on clinical decision-making. These sources include randomized clinical trials, quasi-experimental investigations, correlational studies, field studies, case reports and clinical guidelines based upon professional consensus (4).

As an alternative to weighting individual studies, clinicians may draw upon published syntheses of study findings, typically in the form of systematic reviews and meta-analyses. Hierarchical models of research evidence place both of these methods in the highest tier alongside randomized clinical trials. Systematic reviews evaluate research evidence based upon pre-defined objective criteria. Over the past 10 years, the Cochrane Collaboration and the Agency for Healthcare Research and Quality have accelerated the dissemination of synthesized information related to health care practices through systematic reviews $(5,6)$.

In meta-analyses, the standard methodology for evaluating the strength of evidence involves the calculation of an effect size, or summary statistic showing the magnitude of the treatment effect, averaged across studies. While metaanalyses are a useful metric method for measuring the usefulness of a treatment approach, there are some disadvantages to this technique. Specifically, the use of aggregate estimates of effect size may hide qualitative differences between the individual studies. Additionally, meta-analyses are subject to publication bias (also known as the "file drawer problem"), that is the tendency of publishing studies showing an effect of a treatment rather than those showing no effect, thereby biasing the pool of clinical data from which meta-analyses are conducted.

Nevertheless, synthesized reports on treatment effectiveness, whether in the form of meta-analyses or systematic reviews, remain a valuable resource to inform clinical decision-making.

\section{Clinical expertise}

Two other broad categories of documents that summarize recommendations for translating research evidence into clinical practice are practice guidelines and best practices.

Practice guidelines are "systematically developed statements to assist practitioners and patient decisions about appropriate health care for specific clinical circumstances" (7). These statements are developed through a consensus process that includes clinical and research experts in the appropriate field, and their developers may also elicit input from health care provider organizations, consumer groups and government agencies, depending upon the scope and purpose of the guidelines. In terms of content, practice guidelines may include approaches to the prevention, diagnosis or treatment of an illness (8). Content might be drawn from various theoretical frameworks and flexibility is allowed in the actual implementation of the practice.

Best practices documents aim to guide treatment programme planning and to outline processes that help dissemination of research-based intervention strategies to clinical settings (8). These documents often include guidelines for service delivery, such as recommended scope of services, assessment and intervention techniques, considerations when treating special populations and processes for coordinating treatment with other types of services. In terms of substance use disorders, best practices documents often inform both policy, by describing optimum standards of treatment service delivery for addicted populations, and also the 
advancement of standards for training clinicians in the field of addiction.

\section{WHO Mental Health Gap Action Programme intervention guide}

The WHO Mental Health Gap Action Programme (mhGAP) intervention guide is an important example of the development of a guide based on the best available evidence and through a systematic approach (9). It includes guidance on evidence-based interventions designed to identify and manage a number of priority conditions for lowand middle-income countries, with the objective of scaling up care for mental, neurological and substance use disorders. The mhGAP intervention guide was developed through an intensive process of evidence review. Systematic reviews were conducted to develop evidence-based recommendations. The process involved a WHO Guideline Development Group of international experts who collaborated closely with the WHO Secretariat. The recommendations were then converted into clear stepwise interventions for management of alcohol and substance use disorders, again with the collaboration of an international group of experts.

The mhGAP intervention guide was developed to facilitate mhGAP-related delivery of evidence-based interventions in non-specialized health care settings. However, the recommended interventions included in the sections below also represent the core set of interventions that are needed within a specialized system of care for substance use disorders.

\section{Substance use disorders}

The differences in the neurobiological, physiological, psychological and behavioural effects of the different categories of drugs are substantial. The public health concerns about the effects and consequences of these different substance use disorders and the impact of how the drugs are taken are also substantial. It should be noted that in most parts of the world a single substance use disorder is rare (10). Many individuals who require treatment for substance use are using more than one drug and, in some parts of the world, they are combining their substance use with alcohol. Therefore, when selecting treatment approaches, one should consider polysubstance use/dependence.

\section{Brief interventions}

Brief interventions can be useful with individuals who use small and moderate amounts of drugs and for some specific substance use disorders, such as cannabis use disorders (11). Although brief interventions can also be useful in initially communicating with individuals with serious substance dependence disorders, they are generally not sufficient to help dependent individuals significantly reduce or stop dependent use. However, brief interventions may be a good first step in engaging patients in more intensive treatment.

\section{Harm-reduction strategies}

An overarching harm reduction philosophy should be an essential component to all treatment activities for individuals with substance use disorders. Harm or risk reduction strategies are intended to help patients moderate but not necessarily eliminate harmful behaviours, thereby limiting the physical, psychological and interpersonal harm caused by their drug use. These strategies may constitute the entirety of the interventions used or they may be combined with other treatment strategies.

Regardless, in considering treatments for individuals with substance use disorders, reducing the harms created by drug use should be a main priority. Prevention of drug injection and its harmful consequences, specifically bloodborne diseases, by providing needle and syringe programmes and opioid substitution treatments, facilitating/ providing testing and treatment, when possible, for bloodborne viral illnesses and promoting safe sexual practices are among the main harm reduction approaches. Over time, when a relationship has been established, intensified efforts should be made to encourage people who inject drugs to get more involved in the comprehensive treatment package for their substance use disorders.

\section{Opioid use disorders}

Opioid use disorders are a substantial public health problem. These disorders primarily occur with injection heroin use and use of prescription opiate pain killers (mainly oral use), including oxycodone, hydrocodone, tramadol (increasingly) and buprenorphine (in a few areas). In many parts of the world, injection of heroin is the major contributor to the spread of HIV, hepatitis $\mathrm{C}$ and other infectious diseases $(12,13)$. Overdose deaths associated with both injecting heroin and taking prescribed opiates are a significant concern (14).

The main objectives of treating and rehabilitating people with opioid dependence are to: reduce dependency on illegal drugs and the morbidity and mortality associated with their use; improve physical and psychological health; reduce criminal behaviour; and help social reintegration and functioning. As no single treatment is effective for all individuals with opioid dependence, various psychosocial and pharmacological treatment options are needed. Relapse following detoxification alone is extremely common and therefore detoxification is rarely an adequate treatment on its own. 


\section{Managing opioid overdose and overdose prevention}

Opioid overdose is a potentially fatal event and rapid emergency measures are needed. Naloxone is a short-acting opioid antagonist that can quickly reverse an opioid overdose (15). Naloxone is effective when delivered by intravenous, intramuscular, subcutaneous and intranasal routes (16). Guidelines recommend delivery of naloxone until clinical reversal is apparent.

Naloxone can also be provided to opiate users and family members of individuals who use opioids for use outside of medical settings (e.g. at home) in the event of an opiate overdose. The distribution of naloxone to individuals at risk of overdose (e.g. those being discharged from residential care or those who undergo detoxification) is being increasingly used to prevent fatal overdoses.

\section{Managing opioid withdrawal}

Extensive research on withdrawal from opioids suggests that unless postwithdrawal treatment is effectively implemented, relapse (and potential overdose) is almost inevitable very soon after withdrawal. Where available, opioid agonist maintenance treatment should be considered, with either methadone or buprenorphine as an alternative to withdrawal, and patient education should be conducted about the risks/benefits of withdrawal versus maintenance treatment. If a decision is made about the management of withdrawal symptoms, buprenorphine or methadone should be prescribed for a limited time (e.g. 3 to 21 days) in a low dose with a tapering approach. Care should be taken particularly if the person has been prescribed other sedative drugs. Clonidine or lofexidine can also be given along with other symptomatic treatments (e.g. anti-emetics to treat nausea, simple analgesics to treat pain and light sedatives to treat insomnia).

\section{Opioid relapse prevention pharmacotherapy}

For individuals who have been successfully withdrawn from opioids, naltrexone hydrochloride can be used as part of relapse prevention management. As an opioid antagonist, naltrexone prevents opioid receptors from being activated by agonist compounds, such as heroin or prescribed opioids, and is reported to reduce opioid cravings and prevent relapse. Naltrexone requires that patients be abstinent from opioids for 5-10 days after discontinuation of opioids. Premature use of naltrexone can cause a very uncomfortable immediate withdrawal reaction. Naltrexone is generally taken daily, although a monthly injection of extended release naltrexone may improve patients' adherence to their medication regimens.

\section{Opioid agonist maintenance treatment}

Opioid agonist maintenance treatment (also known as opioid substitution treatment) is defined as the administration under medical supervision of a prescribed long-acting opioid agonist (or partial agonist) medication that has the capacity to prevent the emergence of withdrawal symptoms and reduce craving. These medicines are usually administered orally in the form of a tablet, a film strip or a solution, thereby reducing the risk of infections associated with injections. Medicines used in substitution therapy are prescribed in relatively stable doses over a long period (usually more than 6 months), which allows stabilization of brain functions and prevention of craving and withdrawal. Agonist maintenance therapy has extensive scientific support and is the most effective pharmacological therapy for opioid dependence.

\section{Methadone}

Methadone is a synthetic opioid most commonly used for substitution therapy and is typically administered orally as a liquid. There is a large amount of research that provides very strong support for the safety and efficacy of methadone (16-18). Methadone is included on the WHO Model List of Essential Medicines (19). The initial methadone dose should be based on the level of opioid tolerance, allowing a high margin of safety to reduce inadvertent overdose. The dosage should then be quickly adjusted upwards if there are ongoing opioid withdrawal symptoms and downwards if there is any sedation. From there, the dose should be gradually increased to the point where illicit opioid use stops. Patients should be monitored with clinical assessment and drug testing. Psychosocial assistance should be offered to all patients.

\section{Buprenorphine}

Buprenorphine has a weaker opioid agonist activity than methadone and is not well absorbed if taken orally; therefore, the usual route of administration is sublingual. Because the effect of buprenorphine plateaus with increasing doses, buprenorphine is therefore less likely than either methadone or heroin to cause an opioid overdose, even when taken simultaneously with other opioids. Buprenorphine maintenance treatment should start with a dose that is suited to the pattern of opioid use, including the level of tolerance, the duration of action of opioids used and the timing of the most recent opioid use. From there, the dose should be rapidly increased (i.e. over days) to one that produces stable effects for 24 hours.

\section{Methadone versus buprenorphine for opioid agonist maintenance treat- ment}

Evidence on the effectiveness of methadone and buprenorphine for opioid agonist maintenance treatment shows that both medications provide good outcomes in most cases (20). In general, methadone is frequently recommended over buprenorphine because methadone has been shown to be more cost-effective. However, making both medications available may attract 
greater numbers of people to treatment and improve treatment matching.

\section{Psychosocial treatment}

Psychosocial interventions - including cognitive and behavioural approaches and contingency management techniques — can add to the effectiveness of treatment, if combined with agonist maintenance treatment and medications for assisting opioid management. However, there is little evidence that psychosocial services, in the absence of medications, produce significant benefits for the management of opioid use disorders. Psychosocial services should be made available to all patients, although those who do not take up the offer should not be denied pharmacological treatment.

\section{Psychostimulant use disorders}

Psychostimulant drugs include cocaine and amphetamine-type stimulants (ATS) (known under the names methamphetamine, crystal meth, captagon and yaba). Although there are significant differences in the neurobiological and pharmacokinetic effects between cocaine and ATS (e.g. the half-life of cocaine is 1-2 hours and the half-life of ATS is 10-12 hours), treatment considerations are similar. At the present time, there are no effective medications for maintenance treatment of psychostimulant dependence (21). While medications may be useful in management of the psychosis associated with their acute and chronic use, as well as for the alleviation of symptoms in early abstinence, psychosocial treatments have the best evidence of effectiveness for the treatment of psychostimulant dependence.

\section{Treatment for intoxication/ psychosis and "withdrawal"}

In addition to exhibiting anxiety, depression and psychosis, individuals presenting with acute psychostimulant intoxication may be violent or suicidal (21). Conservative care consists of placing non-threatening individuals in a quiet and calm environment, while more agitated patients may require benzodiazepines or neuroleptics, and possibly gastric lavage and/or activated charcoal to promote clearance of the drug. While traditional antipsychotic medications (e.g. haloperidol) and benzodiazepines are effective in reducing symptoms of psychosis and agitation, olanzapine and other medications appear to be better tolerated.

\section{Harm reduction approaches}

Compared with opioid use disorders, the development of harm reduction strategies for psychostimulant users is much less advanced. In situations where psychostimulants are injected, providing clean injection equipment, education and encouragement to switch to non-injection routes of administration may be useful. In many parts of the world, men who have sex with men use psychostimulants at higher rates than in the general population. The high risk sexual behaviour associated with psychostimulant use is a major contributor to the transmission of HIV. Therefore, easy access to condoms and education about safer sexual practices may be useful harm reduction approaches in this group.

\section{Cannabis use disorders}

Cannabis is the most commonly used illicit drug across the world (22), with an estimated prevalence of 3.9\% (among those aged 15-64) or 180.6 million users. It is estimated that around 1 in 11 recreational cannabis users $(23,24)$ and $25-50 \%$ of daily cannabis users develop cannabis dependency $(25,26)$. Cardiovascular and respiratory function problems have been found to be greater among daily cannabis smokers, and the risk of road traffic incidents while driving under the influence of cannabis is
2-3 times higher $(27,28)$. Cannabis users are twice as likely to develop a mental disorder as non-users, especially if cannabis use started during adolescence and/or there is a predisposition to mental illness. There are no effective medications for cannabis dependence. Psychosocial support is the main treatment option for the management of cannabis dependence, including brief interventions.

\section{Psychosocial treatments for substance use disorders}

The essential factors for the successful use of all psychosocial interventions are empathy, respect for the patient and the use of positive reinforcement methods to encourage and reinforce positive changes in the lives of patients. Praise and support for patient progress (even small increments of change) and consistent support for patients are also critical. There are specific psychosocial counselling approaches that improve treatment success including:

Cognitive behavioural therapy (CBT). This is a form of "talk therapy" based on the principles of conditioning and learning that is used to teach, encourage and support individuals about how to reduce/stop their drug use. CBT encompasses a range of interventions that may be quite different in application and focus and may incorporate relapse prevention and coping skills therapy. CBT provides skills that are valuable in assisting people to reduce drug use and/or start abstaining from drug use, and provides skills to help people sustain their reduced use and/ or abstinence.

Motivational interviewing/motivational enhancement therapy (MI/MET): Many, if not most, problem drug users are unsure about stopping their drug use. MI/MET is a set of techniques that allow professionals treating substance 
use disorders to address the uncertainty common among many drug users and promote changes in their lives $(29,30)$. $\mathrm{MI} / \mathrm{MET}$ helps individuals recognize the problems in their lives created by drug use and create a pathway to make positive changes. When using MI/ MET, health workers respond to patients in a consistent, non-judgmental, supportive and most constructive way by 1 ) expressing empathy; 2 ) developing the difference between the patient's goals and aspirations and his/her current self-destructive behaviour; 3 ) avoiding argument; 4) accommodating the patient's resistance to behaviour change; and 5) supporting the patient's self-efficacy to bring about constructive behaviour changes. MI/MET has broad application in many situations to address the challenges of working with individuals with substance use disorders.

Contingency management/motivational incentives: Contingency management applies the principles of positive reinforcement for performance of desired behaviours consistent with a reduction or cessation of drug use. Typically, contingency management involves the conditional delivery of a voucher (which can be traded for desired items or privileges) or other incentives for behaviours such as attendance at the treatment sessions or production of a negative urine test. Contingency management has been widely applied to many drug dependence disorders, and a meta-analysis of research findings has documented strong evidence of its efficacy in many studies, types of disorders and populations (31).

Family therapy and couples therapy: Family and couples therapy comes from learning-based behavioural models of etiology and uses cognitive-behavioural techniques to bring about change in both maladaptive interpersonal behaviour and problem drug and alcohol use. These techniques use a combination of psychoeducation and CBT to assist the patient and his/her family or spouse in developing more positive coping skills for addressing problem situations that may be associated with drug use. Communication skills are also part of many of these approaches to provide patients and their family/spouse with skills that reduce the maladaptive communication patterns that can lead to relapse to drug use. To effectively deliver family and couples therapy, significant specialized training is needed.

Self-help groups: People with drug dependence can benefit from participating in a self-help group, e.g. Narcotics Anonymous. Involvement in these groups can be very useful in assisting patients to achieve and maintain abstinence from drugs and alcohol. These groups generally recommend a total abstinence from all drugs and alcohol, although in some parts of the world, moderation groups are available.

\section{Implementing treatment approaches}

When decisions are made about which management approaches for substance use disorders should be implemented within a country or region, decisionmakers should consider the priorities of the country and the approaches that would be a good fit for the country. Two of the most important considerations are the use of opioid agonist medicines and the workforce needed for psychosocial treatment.

\section{Opioid agonist medication}

The evidence on the efficacy and effectiveness of methadone and buprenorphine is overwhelming $(20,32)$. Opioid agonist treatment reduces or eliminates illegal opioid use, reduces injection behaviour, reduces relapse and improves health. The training required to prepare doctors to deliver these medicines is relatively simple and the per-patient cost for these pharmacotherapies is less than many other interventions, particularly those involving residential care.
However, one concern about the use of these medicines is their potential diversion to illicit markets. In some areas of the world where these medicines have been introduced with poor controls over prescribing practices and/or lax pharmacy practices, they have been diverted to the illicit market. When the decision is made to consider these medicine, an effective control mechanism is essential.

\section{Psychosocial treatment workforce}

The development of effective treatment systems that can deliver psychosocial treatment is dependent upon the availability of well-trained professionals. In many parts of the world, there are limited opportunities for professionals to receive useful training for effective use of these treatment approaches. Even mental health professionals, including psychiatrists, need some specific training to use psychological interventions for substance use disorders. In addition, there is a shortage of mental health professionals dealing with substance use disorders, particularly in low- and middle-income countries. Therefore, the absence of a qualified, trained workforce is a serious limitation to the delivery of effective psychosocial treatment services.

Workforce training efforts should focus on the substance use disorders of greatest public health concern and build the workforce capacity needed for management of these disorders. One strategy that has been used with success is the use of a pyramid training model, in which highly skilled local experts are trained to provide the foundation for an in-country training capacity. Training physicians and psychiatrists is an essential step to create in-country expert training capacity. In national plans for service implementation, targets for workforce capacity development should be identified to make clear the knowledge and skills needed for training as well as the 
number and type of professionals that will be needed.

\section{Conclusion}

A major challenge to improving the effectiveness of services for substance use disorders is the implementation of evidence-based practices. Efforts have been made, including the WHO mh-GAP intervention guide, to provide reviews and summaries of the existing evidence on the efficacy of a wide range of practices to treat substance use disorders. The treatment of opioid use disorders has received the most empirical attention, and there are numerous medicines and behavioural and harm reduction practices that are established as providing benefits to patients and society. For other substance use disorders, medicines and specific harm reduction strategies have not been as well developed or researched. However, there are a set of psychosocial and behavioural strategies that have evidence to support their usefulness. The summary provided in this article provides a snapshot of the evidence-based practices for substance use disorders as of 2016. As new evidence arises, this document and others like it will need to be updated to maintain an accurate picture of the knowledge available to guide the development of services for substance use disorders.

\section{Funding: None.}

Competing interests: None declared.

\section{References}

1. Lamb S, Greenlick MR, McCarty D, editors. Bridging the gap between research and practice: Forging partnerships with community-based drug and alcohol treatment. Washington (DC): National Academy Press; 1998.

2. Sackett DL, Straus SE, Richardson WS, Rosenberg W, Haynes RB. Evidence-based medicine: How to practice and teach EBM. London: Churchill Livingstone; 2000.

3. Sackett DL, Wennberg JE. Choosing the best research design for each question. BMJ. 1997;315:1636.

4. Miller WR, Zweben J, Johnson WR. Evidence-based treatment: Why, what, where, when, and how? J Subst Abuse Treat. 2005;29:267-76.

5. Clarke M. The Cochrane collaboration and systematic reviews. Br J Surg. 2007;94:391-2.

6. Walshe K, Rundall TG. Evidence-based management: From theory to practice in health care. Milbank Q. 2001;79:429-57.

7. Field MJ, Lohr KN, editors. Clinical practice guidelines: Directions for a new program. Institute of Medicine Committee to Advise the Public Health Service on Clinical Practice Guidelines. Washington (DC): National Academy Press; 1990.

8. Lacroix SI. Evidence-based and best practice addiction treatment resources: A primer for librarians. Behav Soc Sci Librar. 2002;21:59-72.

9. World Health Organization. WHO Mental Health Gap Action Programme (mhGAP). 2015 (http://www.who.int/mental_ health/mhgap/en/, accessed 10 April 2017).

10. World drug report - 2014. New York: United Nations Office on Drugs and Crime (UNODC); 2014 (http://www.unodc.org/ documents/wdr2014/World_Drug_Report_2014_web.pdf, accessed 10 April 2017).

11. Babor TF, McRee BG, Kassebaum PA, Grimaldi PL, Ahmed K, Bray J. Screening, brief intervention, and referral to treatment (SBIRT): Toward a public health approach to the management of substance abuse. Subst Abus. 2007;28(3):7-30.

12. Bao YP, Liu ZM. Systematic review of HIV and HCV infection among drug users in China. Int J STD AIDS. 2009;20(6):399405. 10.1258/ijsa.2008.008362.

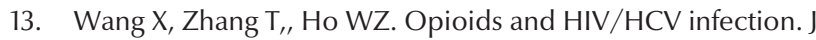
Neuroimmune Pharmacol. 2011;6(4):477-89. 10.1007/s11481011-9296-1.
14. Hser Y-I, Evans E, Grella C, Ling W, Anglin D. Long-term course of opioid addiction. Harv Rev Psychiatry. 2015;23(2):76-89. 10.1097/HRP.0000000000000052.

15. Boyer E. Management of opioid analgesic overdose. N Engl J Med. 2012;367:146-55

16. SAMHSA opioid overdose prevention toolkit. HHS Publication No. (SMA) 13-4742. Rockville, MD: Substance Abuse and Mental Health Services Administration; 2013 (https:// store.samhsa.gov/shin/content/SMA13-4742/Overdose_ Toolkit_2014_Jan.pdf, accessed 10 April 2017).

17. Amato L, Davoli M, Perucci CA, Ferri M, Faggiano F, Mattick RP. An overview of systematic reviews of effectiveness of opiate maintenance therapies: Available evidence to information clinical practice and research. J Subst Abuse Treat. 2005;28:321-9.

18. Mattick RP, Breen C, Kimber J, Davoli M. Methadone maintenance therapy versus no opioid replacement therapy for opioid dependence. Cochrane Database Syst Rev. 2009 Jul 8;(3):CD002209. 10.1002/14651858.CD002209.pub2.

19. WHO model list of essential medications. Geneva: World Health Organization; 2013. (http://www.who.int/medicines/publications/essentialmedicines/18th_EML_Final_ web_8Jul13.pdf, accessed 10 April 2017).

20. Connock M, Juarez-Garcia A, Jowett S, Frew E, Liu Z, Taylor $\mathrm{RJ}$, et al. Methadone and buprenorphine for the management of opioid dependence: A systematic review and economic evaluation. Health Technol Assess. 2007;11(9):1-171.

21. Ling W, Mooney L, Haglund M. Treating methamphetamine abuse disorder. Curr Psychiatr. 2014;13(9):37-44.

22. World drug report - 2013. New York: United Nations Office on Drugs and Crime (UNODC); 2013 (http://www.unodc.org/ unodc/secured/wdr/wdr2013/World_Drug_Report_2013. pdf, accessed 10 April 2017).

23. Anthony J, Warner LA, Kessler RC. Comparative epidemiology of dependence on tobacco, alcohol, controlled substances, and inhalants: Basic findings from the National Comorbidity Survey. Exp Clin Psychopharmacol. 1994;2:244-68.

24. Lopez-Quintero C, Pérez de los Cobos J, Hasin DS, Okuda M, Wang S, Grant BF, et al. Probability and predictors of transition from first use to dependence on nicotine, alcohol, cannabis, and cocaine: Results of the National Epidemiologic Survey on Alcohol and Related Conditions (NESARC). Drug Alcohol Depend. 2011;115(1-2):120-30. 
25. Hall W. The adverse health effects of cannabis use: What are they, and what are their implications for policy? Int J Drug Policy. 2009;20:458-66.

26. Hall W, Degenhardt L. Adverse health effects of non-medical cannabis use. Lancet. 2009;374:1383-91.

27. Hall W. What has research over the past two decades revealed about the adverse health effects of recreational cannabis use? Addiction. 2015;110(1):19-35. 10.1111/add.12703

28. Ramaekers JG, Berghaus G, van Laar M, Drummer OH. Dose related risk of motor vehicle crashes after cannabis use. Drug Alcohol Depend. 2004;73(2):109-19.
29. Burke BL, Arkowitz H, Menchola M. The efficacy of motivational interviewing: A meta-analysis of controlled clinical trials. J Consult Clin Psychol. 2003;71:843-61.

30. Hettema J, Steele J, Miller WR. Motivational interviewing. Annu Rev Clin Psychol. 2005;1:91-111.

31. Prendergast M, Podus D, Finney J, Greenwell L, Roll J. Contingency management for treatment of substance use disorders: A meta-analysis. Addiction. 2006;101(11):1546-60.

32. National Institute of Health and Clinical Excellence. 2011. Drug misuse: psychosocial interventions: NICE guideline 51 [webpage] (http://guidance.nice.org.uk/CG51, accessed 10 April 2017). 